\title{
Editorial - The growing inter-disciplinary field of longitudinal studies beginning in early life: this journal's response
}

\author{
Michael Wadsworth, Carol Dezateux \\ Health Section Editors
}

Longitudinal studies concerned with development and health in childhood were in important respects the springboard for a life course approach to health, educational, developmental and policy studies. Early examples of longitudinal studies of children include, in the United States, the Terman study of intelligence (1921), the Berkeley Guidance Study (1928), the Fels Longitudinal Study of child growth (1929), the Oakland Growth Study (1931), and the Cambridge-Somerville Study of delinquency (1939). Early European examples include the 1932 Scottish Mental Survey of cognitive function of all school children, the British Boyd-Orr study (1937-9) of diet and growth, and the Swedish (Malmo) Children of the 1930s study (1938). All of these studies from the inter-war period were developed by later generations of researchers as resources for the longitudinal study of adult outcomes, in terms of, for example, mental and physical health, cognitive function, educational development and behavioural trajectories starting in childhood.

Then immediately after the Second World War concerns about perinatal and infant mortality and reproductive health led to the first national British birth cohort study. Begun in 1946, two years before the UK National Health Service, the National Survey of Health and Development study identified the associated range of risk factors and the magnitude of socio-economic and geographic inequity in the distribution of infant deaths and, at follow-up, in physical and mental growth and development during childhood. Subsequently, two further British birth cohort studies were begun in 1958 (the National Child Development Study) and 1970 (the 1970 British Birth Cohort), initially to find out whether these markers of perinatal and infant health had improved in the context of a universal National Health Service free at the point of use: their initial findings profoundly influenced UK maternity services. Each of these three studies went on to collect data on cognitive development, behaviour, family life and educational attainment throughout childhood and adolescence, and so provided unique new opportunities for research into human development, new policy perspectives on education, family life and child care, and new understanding about the impact of socio-economic change on children. Their continuation into adulthood provides exceptional opportunities for modelling the profiles of individual development and change in ways that were not anticipated when they commenced. Parallel developments in Sweden included the Individual Development and Adaptation programme starting in 1964 and the Project Metropolitan longitudinal studies based in the Nordic capital cities, including the Stockholm study based on male births followed up from 1953.

The methods and hypotheses of those studies were developed further by subsequent longitudinal studies which probed deeper into the processes of early life growth and development starting in pregnancy (for example the Avon Longitudinal Study of Parents and Children (1991-2), and the Northern Finland Cohort Studies (1966 and 1986) or at birth (e.g. in New Zealand, the Dunedin Multidisciplinary Health and Development Study in 1972-73 and the Christchurch, Health and Development Study in 1977). These studies were more intense in their frequency of data collection and the extent of data collected in early life and childhood, and made good use of new methods of measurement in the social, psychological and health sciences. A comprehensive list of longitudinal studies up to 2006 can be found in http://www.longviewuk.com/pages/documents/Re viewofPanelandCohortStudies-

Appendix27.04.06.pdf.

All of these studies from the pre- and post-war periods continue to contribute greatly to understanding the life-long impact of early life physical and mental development and its family and 
socio-economic context. They stimulate new thinking about early life, and continue to provide unique research opportunities for exploring how early life growth and developmental effects are mediated and modified with age. They ask, for example, how aspects of the child's socio-economic and material environment affect biological, cognitive and behavioural development, growth, and the onset of physical and mental illnesses. They are now evaluating childhood and adult trajectories and the accumulation of risk in relation to a wide range of factors including, for instance, socioeconomic position and the processes of ageing. Data from these same sources have also been used in comparative studies of cohort differences of early life and childhood growth, and cognitive and physical developmental trajectories, behaviour and attainment, and adult health and health-related behaviour, making opportune use of cohort differences in exposure to environmental and socioeconomic differences of all kinds.

Some studies collect biological samples, adding genetic information derived from their cohort members, while a number of the longer running studies are undertaking research into the health, educational and occupational attainment of their cohort's offspring, thereby creating an intergenerational data resource. In addition, these latter kinds of questions are being addressed in longitudinal studies of households which have collected data on panel members' children followed up from birth (e.g. the US Panel Study of Income Dynamics (1998), Understanding Society (2009), and the German Socio-economic Panel Study (1990)), and children of longitudinal studies of adolescent cohorts (e.g. the US National Longitudinal Study of Youth study (1979)), and in Europe, the Longitudinal Study of Immigrants to Germany (1984). These studies are continuing to stimulate new thinking about childhood and early life in all the relevant research disciplines as well as among policymakers. An exciting development is the comparison between cohorts in countries of different income status and with contrasting socioeconomic patterning of exposures and behaviours.

In the health sciences new ideas are developing about the processes by which environmental and genetic effects combine to influence health and behaviour throughout the life course. New methods of measurement (e.g. of an individual's and family's genetic makeup, organ development in the prenatal and early postnatal period, behaviours such as physical activity, environmental exposures, and agerelated change - both developmental and in adulthood - in for example cardiac, respiratory or cognitive function) make it possible to study the influence of genetically-determined processes on trajectories of development and ageing, and - most recently - to increase our understanding of how environmental exposures, both adverse and protective, are expressed through epigenetic mechanisms.

Such studies, especially those involving research on gene-environment interaction, require very large samples, ideally beginning before or soon after birth (e.g. the Danish National Birth Cohort (1996), the Norwegian Mother and Child Cohort Study (1999), the UK Millennium Cohort study (2000-2001), the French L'Étude Longitudinale Française depuis l'Enfance study (2012), and in the UK the new national birth cohort called the 'Life Study' (2013), as well as before pregnancy, such as in the US National Children's Study (2012) and, in England, the Southampton Women's Survey (1998). Many smaller sample longitudinal studies have been established including those, such as the Rotterdam-based Generation R study (2006) and the Born in Bradford Study (2010), designed to study specific groups, exposures, conditions and/or outcomes.

Throughout their histories the early longitudinal studies have also stimulated new thinking in the behavioural and social sciences concerned, for instance, with children's well-being, and the life course and inter-generational development of, for example, pro- and anti-social behaviour, the processes of learning, and the effectiveness of policies for child care and education. Such work has extended to the impact of rapid social and economic change across time and generations on children's lives, using cross-cohort comparative research. New and recent longitudinal studies in these areas include the Millennium Cohort study (2000-2001) based on births in 400 polling districts the Young Lives project comprising new child cohort studies in Ethiopia, India, Peru and Vietnam and the multi-cohort German National Education Panel study (2008) that for the youngest of 6 age cohorts extends data collection back to birth. These new ideas in the social and behavioural sciences have also generated policy-oriented 'Growing Up' studies such as Growing Up in Australia (2004), Growing Up 
in Scotland (2004), Growing Up in Ireland (2007) and Growing up In New Zealand (2009-2010). The European studies listed, together with the ELFE, ALSPAC, Generation R and Born in Bradford studies - all of which have a major interest in child health form the European Child Cohort Studies Network (EUCCONET), which extends internationally to include such studies as the Young Lives project and Growing Up in New Zealand (2010). Details of all the EUCCONET studies can be found via www.eucconet.com.

The social, behavioural and population health sciences concerned with childhood are all benefiting from new methods of measurement in longitudinal studies (e.g. of early cognitive function, nutrition in childhood, biomarkers, child rearing practices, environmental pollutants). In addition, research across the disciplines is stimulating new development and application of more sophisticated methods of data analysis, including multi-level or latent class models, methods of handling missing data such as multiple imputation, as well as Mendelian randomisation.

The value of longitudinal studies of early life and childhood is being increasingly recognised by policy makers, governments, professionals concerned with children, and research funders, as well as the public. Consequently the older studies are being continued and expanded, and new funding invested in many countries in newer larger studies. This investment is encouraging researchers to make use of data from the older as well as the more recent life course studies and of studies carried out in different settings. For example data are being shared, harmonised and pooled through initiatives such as ENRIECO (Environmental Risks in European Birth Cohorts www.enrieco.org), CHICOS (Developing a Child Cohort Research Strategy for Europe www.chicosproject.eu), I4C (International Childhood Cancer Consortium

http://ije.oxfordjournals.org/content/36/4/724.full), GA2LEN (Global Allergy and Asthma European Network www.ga21en.net), and CLOSER (Cohorts and Longitudinal Studies Enhancement Resource www.closer.ac.uk). There is also increasing prospective harmonisation of data on key variables and collection plans, such as the HALCyon collaborative research programme funded by five UK Research Councils under the New Dynamics of Ageing programme, the remit of which is to improve the quality of life of older people. (see http://www.halcyon.ac.uk/?q=project )

Longitudinal and Life Course Studies offers significant benefits to researchers and academics wishing to publish their work in these expanding and fast developing areas of research in early life and childhood. This editorial announces the widening of our editorial team to increase the research expertise available in the child health and population health sciences while recognising the distinctive feature of early childhood study. The expansion signals our desire to publish reports of cross-disciplinary research using longitudinal data from different times or settings to study influences on health and cognitive and behavioural development, which occur during the period from conception to early adolescence and across generations, and their impact and effects throughout the life course. We also wish to publish research that evaluates or otherwise impacts on policy and practice, crosses disciplinary boundaries and/or reports methodological advances in data collection and/or data analysis relevant to longitudinal studies.

Key advantages for authors include:

- international and multi-disciplinary readership

- anonymous peer review

- policy of open access

- speed of publication

- encouragement of inter-disciplinary papers

- acceptance of longer papers than customary, normally up to a maximum of 7,000 words

- publication of papers reporting original research and methodological developments, shorter research notes, profiles of cohort studies, expert tutorials on methodological topics, and abstracts from the annual meeting of the Society for Longitudinal and Life Course Studies (www.longstudies.longviewuk.com).

We look forward to receiving manuscripts for publication. 